\title{
Transcatheter repair of a left ventricular outflow tract pseudoaneurysm following composite aortic root replacement using an Amplatzer Septal Occluder
}

\author{
Cynthia Kiang ${ }^{1}$, Praveen Sridhar ${ }^{1}$, Karl Karlson ${ }^{1}$, Robert Eberhardt ${ }^{1}$, Niloo Edwards ${ }^{1}$, \\ Meaghan Doucette ${ }^{1}$, and Nikola Dobrilovic ${ }^{1}$ \\ ${ }^{1}$ Boston Medical Center
}

June 15, 2020

\begin{abstract}
The emergence of endovascular techniques has enabled minimally invasive options for repair and salvage of postoperative sequelae from complex aortic operations. There have been few reports of percutaneous transcatheter repair of aortic pseudoaneurysms after composite aortic root replacement with reports of only two patients with follow-up longer than two years. We report a case of a 35 year man who developed an aortic root pseudoaneurysm following a composite aortic root replacement and was successfully managed by transcatheter repair using an Amplatzer Septal Occluder without evidence of recurrence at two year follow-up.
\end{abstract}

\section{Case Report}

The patient is a 35 year-old man with Type II Diabetes Mellitus, hypertension, hyperlipidemia, and morbid obesity (BMI 37) who presented in 2009 with a symptomatic $7.7 \mathrm{~cm}$ ascending aortic aneurysm. He had no prior history of cardiac disease, no family history of connective tissue disorders and no history of aortopathy. At his initial presentation, the patient presented with severe palpitations and was found to have severe aortic insufficiency (AI) with an annular diameter of $33 \mathrm{~mm}$ and preserved left ventricular (LV) function. He underwent a "Modified Bentall" composite aortic root replacement via median sternotomy and cardiopulmonary bypass[1]. Arterial cannulation was achieved via the femoral artery and right atrium, retrograde cardioplegia was administered via the coronary sinus and the LV was vented via the right superior pulmonary vein. The aorta was cross-clamped proximal to the innominate artery. A \#31 composite St. Jude mechanical valve conduit was used. The proximal anastomosis was completed using interrupted sutures while the distal anastomosis was performed using a running suture repair with pledgeted reinforcement. Coronary buttons were fashioned and anastomosed to the graft conduit. An intraoperative transthoracic echocardiogram (TEE) showed no paravalvular leak. The patient had an unremarkable postoperative course and was progressing well at follow-up.

Ten months post-procedure the patient presented to the emergency department with complaints of chest pain. Imaging with a computed tomography with arterial phase contrast (CTA) revealed contrast extravasation at the level of the aortic annulus suggesting an anastomotic pseudoaneurysm; however, transthoracic echocardiography (TTE) at this time did not show evidence of flow into this space (Figure 1). His symptoms resolved and no intervention was undertaken at this time. The patient was followed with serial CTAs.

Eight years following his repair, our patient was found to have pseudoaneurysm enlargement on surveillance CTA from his prior imaging, and again endorsed symptoms of intermittent chest pain (Figure 2). He underwent TEE which the previously identified pseudoaneurysm at the level of the proximal anastomosis in the 
interatrial septum. Left heart catheterization was subsequently performed and confirmed the extravasation of contrast proximal to the mechanical valve and into the pseudoaneurysm space (Figure 3).

The patient was discussed in multidisciplinary conference including cardiology, interventional cardiology, cardiac surgery, and anesthesiology. Re-operation for pseudoaneurysm repair was weighed against percutaneous endovascular repair and discussed with the patient and the decision was made to pursue percutaneous closure of the defect.

The procedure was performed under general anesthesia with intraoperative TEE guidance. Two transseptal punctures were performed for angiography and for device deployment via an 8.5-Fr sheath in the right femoral vein. The pseudoaneurysm space was accessed using a 6-Fr glide catheter and angled glidewire. The defect size was measured approximately $10 \mathrm{~mm}$. A $14 \mathrm{~mm}$ Amplatzer ${ }^{\mathrm{TM}}$ Vascular Plug II (Abbott Laboratories; Chicago, IL) was placed, however persistent leak was noted. An $8 \mathrm{~mm}$ Amplatzer ${ }^{\mathrm{TM}}$ Septal Occluder (Abbott Laboratories; Chicago, IL) device was then deployed. This resulted in a stable position of the plug and moderately improved the leak by color flow and angiography. TEE confirmed residual but significantly improved shunt flow. The procedure was concluded and the patient was admitted postprocedurally for observation. A TEE was repeated on post procedure day one and showed the vascular plug near in appropriate position with residual trace AI observed. The patient recovered uneventfully and underwent repeat TTE at 3 month follow-up.

TTE at follow-up revealed no obvious AI in the area of the device and the previous pseudoaneurysm was no longer evident. The patient also underwent a follow-up CTA at that time and the previously seen extraluminal contrast collection was no longer present. Specifically, the previously seen pseudoaneurysm abutting the aortic root had decreased in size and thrombosed (Figure 4). The patient has resumed regular activity and remains free of recurrence two years following his intervention.

\section{Discussion}

Endovascular technologies are increasingly available to clinicians as options for minimally invasive approaches to complex pathologies [2-6]. While an anastomotic pseudoaneurysm following aortic root replacement is a rare sequela, it poses a challenge given the added morbidity and potential mortality of a reoperative sternotomy [7,8]. The Amplatzer ${ }^{\mathrm{TM}}$ Septal Occluder has been previously used in a limited fashion for percutaneous closure of aortic pseudoaneurysms in 13 cases, of whom only two had prior aortic root replacements $[5,6]$. While there is some evidence that transcatheter approaches may not be effective, we present a clin-

ical scenario of a postoperative pseudoaneurysm arising from the left ventricular outflow tract (LVOT) of a patient who had previously undergone a composite root replacement [3]. Given the morbidity and potential mortality of an open repair, the patient underwent a successful percutaneous transcatheter repair with radiographic resolution seen two years following the intervention. This case adds to recent literature suggesting that alternative techniques to open repair may be safe and durable for the salvage of composite root replacements with pseudoaneurysmal complications. While the transcatheter repair has been limited to patients who are truly not operative candidates, it can be a useful tool to employ depending upon the resources available to the clinician and preferences of the patient.

\section{Figure Legend}

Figure 1 - Arterial phase Computed Tomography (CTA) in the axial view at initial diagnosis of pseudoaneurysm. (A) Right main coronary ostium, (B) Left main coronary ostium, (C) Pseudoaneurysm with contrast extravasation (arrow), (D) Pseudoaneurysm in largest dimension arising from the left ventricular outflow tract (LVOT) (arrow).

Figure 2 - CTA, axial images, demonstrating interval growth of pseudoaneurysm. (A) Right main coronary ostium, (B) Left main coronary ostium, (C) Pseudoaneurysm with contrast extravasation (arrow), (D) Pseudoaneurysm in largest dimension arising from the left ventricular outflow tract LVOT (arrow).

Figure 3 - Left heart catheterization via transseptal puncture revealing contrast extravasation from the LVOT proximal to the replacement aortic valve. 
Figure 4 - CTA, axial images, demonstrating resolution of previously documented pseudoaneurysm. (A) Right main coronary ostium, (B) Left main coronary ostium, (C) Thrombosed pseudoaneurysm sac, (D) Thrombosed pseudoaneurysm with no contrast extravasation from the LVOT.

\section{References}

[1] Bentall H, De Bono A. A technique for complete replacement of the ascending aorta. Thorax 1968;23:3389. doi:10.1136/thx.23.4.338.

[2] Hao F, Genshaft S, Kee ST, Canan T, Yang EH, Moriarty JM. Percutaneous Transthoracic Treatment of Ascending Aortic and Root Pseudoaneurysms: Procedural Aspects and Guidance with the Use of Multimodality Imaging. J Vasc Interv Radiol 2018;29:628-31. doi:10.1016/j.jvir.2018.01.767.

[3] Hibino M, Katada Y, Shibayama K, Obunai K, Watanabe H, Kawano Y, et al. Recurrent aortic root pseudoaneurysm after transcatheter occlusion-A word of caution. J Card Surg 2018;33:190-3. doi:10.1111/jocs.13568.

[4] Yin WH, Wei J, Tsai SK, Hsiung MC, Lee YT, Yu HP, et al. Transcatheter intervention for complex ascending aortic pseudoaneurysm after cardiac surgery. Circ J 2014;78:2215-8. doi:10.1253/circj.CJ-14-0154.

[5] Kanani RS, Neilan TG, Palacios IF, Garasic JM. Novel use of the Amplatzer@ septal occluder device in the percutaneous closure of ascending aortic pseudoaneurysms: A case series. Catheter Cardiovasc Interv 2007;69:146-53. doi:10.1002/ccd.20794.

[6] Steinberg ZL, Don CW, Sun JCJ, Gill EA, Goldberg SL. Percutaneous Repair of Aortic Pseudoaneurysms: A Case Series. J Invasive Cardiol 2016;28:E6-10.

[7] Mulder EJ, Van Bockel JH, Maas J, Van Den Akker PJ, Hermans J. Morbidity and mortality of reconstructive surgery of noninfected false aneurysms detected long after aortic prosthetic reconstruction. Arch Surg 1998;133:45-9. doi:10.1001/archsurg.133.1.45.

[8] Maroto LC, Carnero M, Cobiella J, García M, Vilacosta I, Reguillo F, et al. Reoperation for composite valve graft failure: Operative results and midterm survival. J Card Surg 2018;33:330-6. doi:10.1111/jocs.13710.

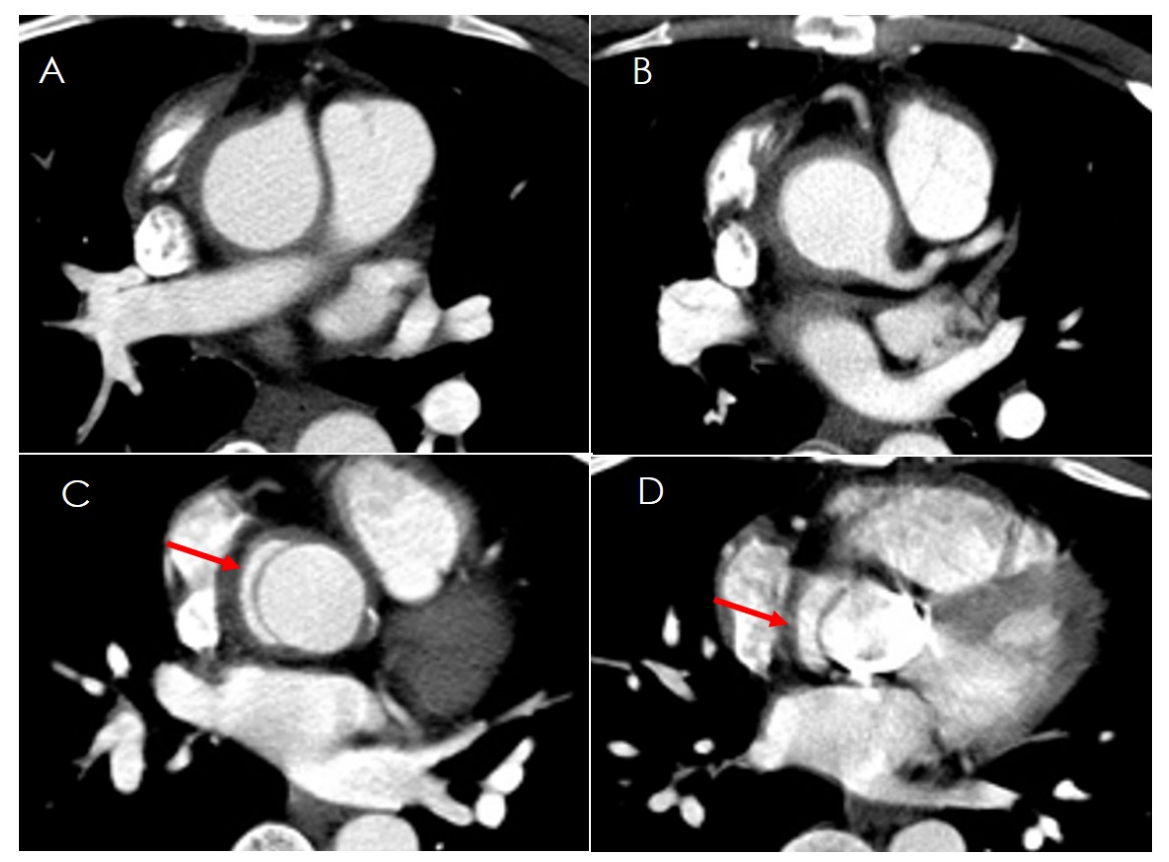



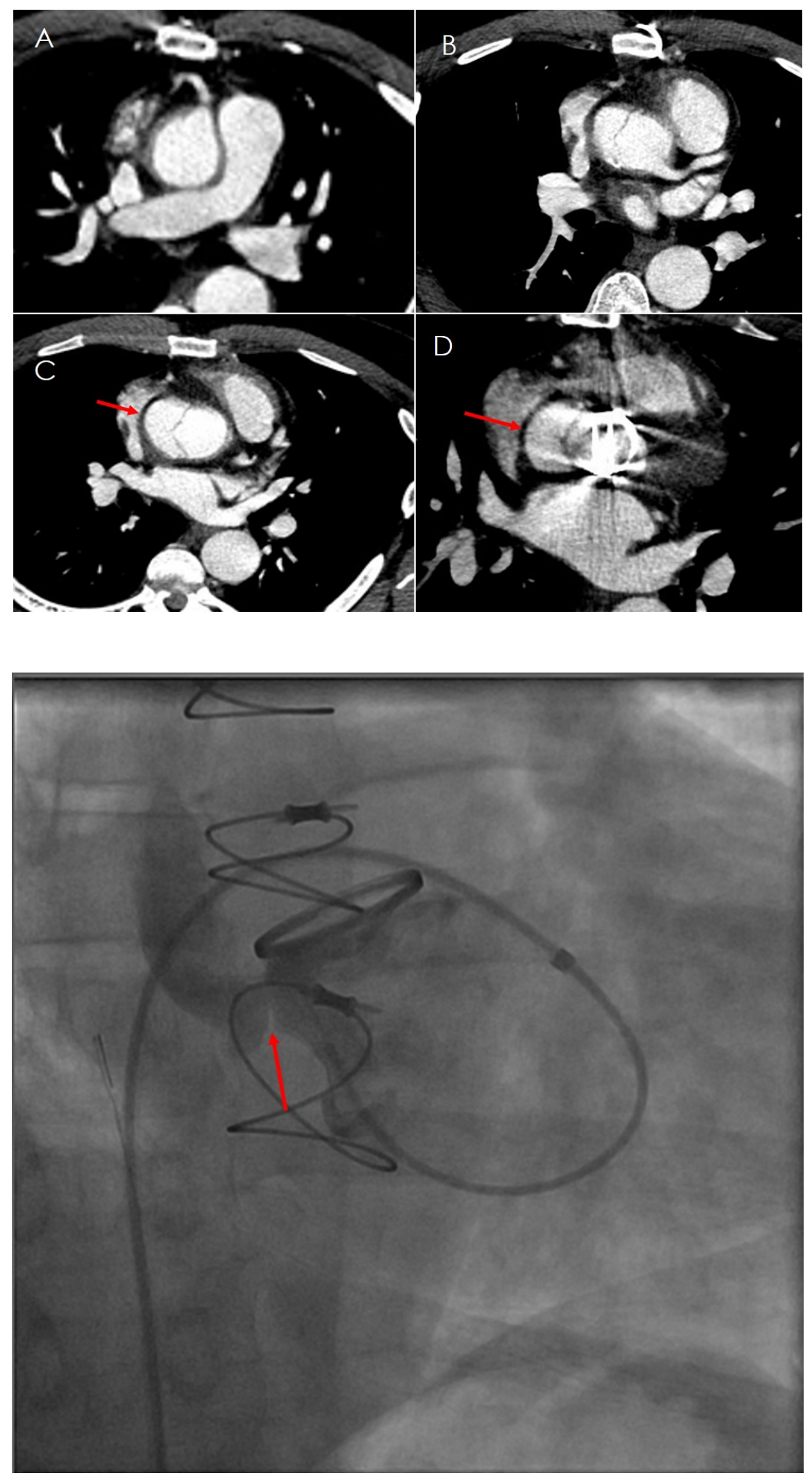


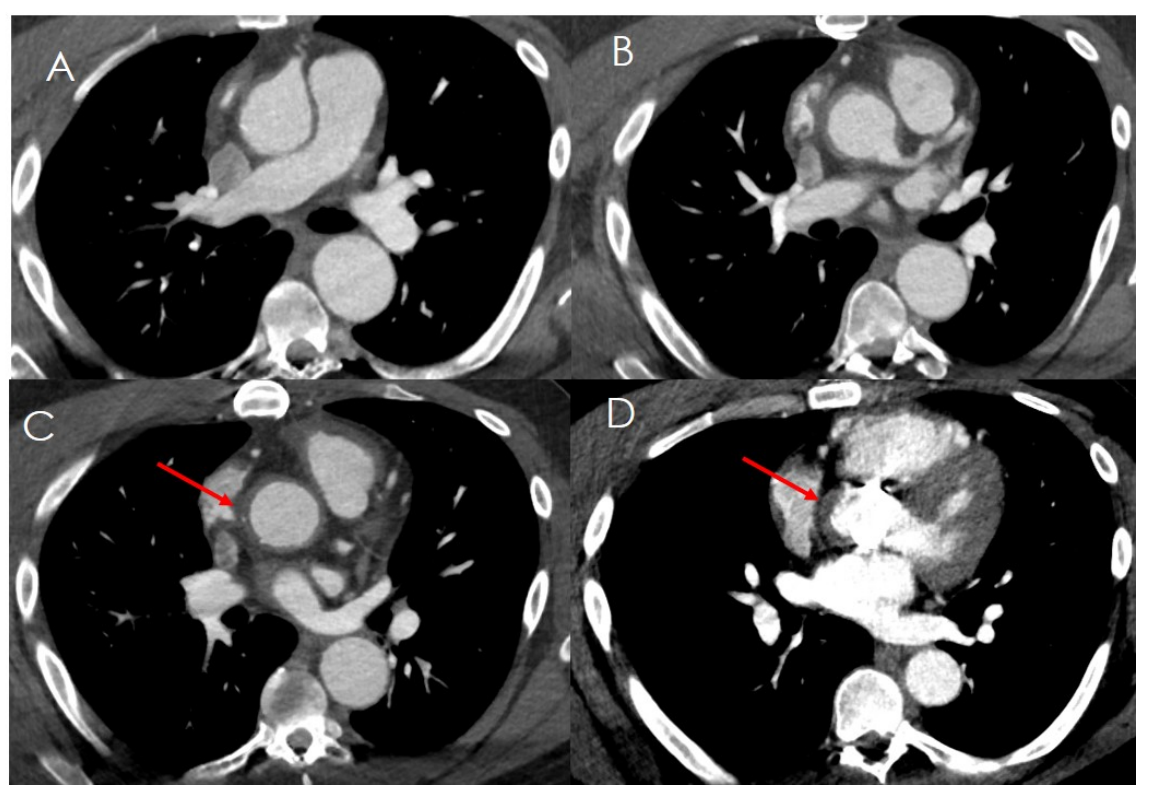

